Contributions to Game Theory and Management, XIII, 207-217

\title{
Nash Bargaining Solution as Negotiation Concept for Resource Allocation Problem: Analysis of Experimental Data *
}

\author{
Nikolay A. Korgin and Vsevolod O. Korepanov
}

\author{
V.A. Trapeznikov Institute of Control Sciences of Russian Academy of Sciences \\ Profsoyuznaya st. 65, Moscow, 117997, Russian Federation \\ E-mail: nkorgin@ipu.ru, vkorepanov@ipu.ru
}

\begin{abstract}
Motivated by research works on Zeuthen-Hicks bargaining, which leads to the Nash bargaining solution (Vetschera, 2018), we analyze data obtained during experimental resource allocation gaming with Yang-Hajek's mechanism from the class of proportional allocation mechanisms. Games were designed in the form of negotiation to allow players to reach consensus. Behavior models based on best response, constant behavior, and Nash bargaining solution are defined. Analysis conducted over decisions made by participants shows that a significant share of all decisions leads to an increase of Nash bargaining value. It is even higher than the share of decisions that are in agreement with the best-response concept. Consensus-ended games show more but subtle attraction to Nash bargaining solution behavior. We discuss how these decisions correspond with other types of behavior actively exhibited by participants of this experiments - so-called constant behavior and with the end of negotiation process in games.
\end{abstract}

Keywords: resource allocation mechanisms, Nash implementation, Nash bargaining solution.

\section{Introduction}

Motivated by research works on Zeuthen-Hicks bargaining, which leads to the Nash bargaining solution (Harsanyi, 1956); Vetschera, 2018), we analyze data obtained during experimental gaming comparison of resource allocation rules in case of transferable utilities described in (Korgin and Korepanov, 2017). In that research, several mechanisms were compared in setting with quasi-linear utilities: a mechanism (YH) from the class of proportional allocation mechanisms (Yang and Hajek, 2005; Başar and Maheswaran, 2003), a mechanism (GL) with balanced payments using the Groves-Ledyard rule (Groves and Ledyard, 1977) that gives the efficient solution of the problem as a Nash equilibrium in the players' game introduced in (Korgin, 2016), as well as its modification (GLR) reducing the dimension of the action space of the players (Korgin, 2016) and a mechanism based on distributed optimization algorithm (ADMM) problem (Boyd and Vandenberghe, 2004).

We analyze decisions made by participants in games with YH mechanism. We show that a significant share of all decisions leads to an increase of Nash bargaining value, and it is even higher than the share of decisions that are in agreement with the best-response concept.

We discuss how these decisions correspond with other types of behavior actively exhibited by participants of these experiments - so-called constant behavior and with the end of negotiation process in games.

\footnotetext{
* This work is partially supported by the RFBR, project no. 19-29-07525 and by the RSF, project no. 16-19-10609.
} 


\section{Resource Allocation Problem}

An organizational system consists of a single Principal and a set $N=\{1, \ldots, n\}$ of players. Principal disposes of some infinitely divisible good in a limited amount $R \in \mathbb{R}^{1}$ and allots it among the players in any proportion.

The utility of each player $i \in N$ in terms of the good $x_{i} \in[0, R]$ allotted to him is described by a function $u_{i}(\bullet): \mathbb{R}^{1} \rightarrow \mathbb{R}^{1}$ belonging to a certain set $U_{i}$ of admissible utility functions.

The set of admissible allocations is

$$
A=\left\{x=\left(x_{1}, \ldots, x_{n}\right): \sum_{i \in N} x_{i} \leq R, x \in \mathbb{R}_{+}^{n}\right\},
$$

the set of possible utility profiles is

$$
U=\left\{u=\left(u_{1}(\bullet), \ldots, u_{n}(\bullet)\right): u_{i}(\bullet) \in U_{i}, i \in N\right\} .
$$

The problem is to find such an allocation mapping $g(\bullet): U \rightarrow A$ that is efficient in the sense that it maximizes the total utility of all players for any utility profile $u \in U$, i.e.,

$$
g(u) \in \underset{x \in A}{\operatorname{Arg} \max } \sum_{i \in N} u_{i}\left(x_{i}\right) .
$$

\section{3. $\quad$ Model}

We consider model $N=\{1,2,3\}$ with utility functions $u_{i}\left(x_{i}\right)=\sqrt{r_{i}+x_{i}}$, where $r=(1,9,25)$ - profile of "initial endowment" of players or, generally, types of utility functions. Type $r_{i}$ of a player $i$ is private information and generally not known to the Principal. Amount of disposable resource is $R=115$.

An efficient allocation accordingly to the right part of (3) is allocation when sum of utilities attain maximum value. For our model, efficient allocation and profit of each player:

$$
\begin{aligned}
& x^{e f f}=(49,41,25) \\
& u^{\text {eff }}=u_{1}\left(x_{1}^{e f f}\right)=u_{3}\left(x_{3}^{e f f}\right)=u_{3}\left(x_{3}^{e f f}\right) \approx 7.07
\end{aligned}
$$

\subsection{Incentive Incompatibility}

For common case of types $r$ the efficient allocation is determined according to:

$$
x_{i}=\left(R+\sum_{i \in N} r_{i}\right) / n-r_{i}, i \in N .
$$

Obviously, being answered about $r_{i}$ any player strives for underrating the value of this parameter (to increase his utility) instead of truth-telling. Thus, each player $i$ answer $r_{i}=1$, "efficient allocation" will be $x^{*} \approx(38.3,38.3,38.3)$, but total utility with real players' types will be less than optimal since $x^{*} \neq x^{\text {eff }}$.

So we have the incentive incompatibility problem.

\subsection{Game Process Model and Experimental Data}

The Game process is implemented in the form of an iterative process as follows. At each iteration (step), $n$ bids (one bid from each player) are acquired and processed according to the rules of the Yang-Hajek resource allocation mechanism (see 
appendix 1). The result of its operation is reported to all players. At the next step, any player may vary his bid, possibly affecting the result. The game process stops if none of the players varies his bid ("stop rule"), or the process reaches a maximum admissible step $T$ known to all players. Parameter $T$ was different in some game sessions: 60,20 , or 15 . The payoff of each player is defined as the profit at the last step.

The last step is crucial because players receive payoffs according to the $\mathrm{YH}$ mechanism on the last step. At the same time, the "stop rule" (none of the players varies his bid) allows players to reach an agreement.

The participants of experiments were Russian students of several State Universities of Moscow, Perm, Samara cities from faculties of Technology or Economics. Participants of one session studied the game rules and play in learning games, and then they were randomly allocated in groups of three and play final (test) games. We treat the results of each group as one separate game.

Notations: at each step $t$ players make bids $s_{1}(t), s_{2}(t), s_{3}(t)$ - bids of the first, second and third player accordingly. The situation at step $t$ is the tuple $s(t)=$ $\left(s_{1}(t), s_{2}(t), s_{3}(t)\right)$. Then, in accordance with the YH mechanism, they receive resources $x(s(t))$, give transfers $\tau(s(t))$ and their profits are $\phi(s(t))=u(x(s(t))-$ $\tau(s(t))$.

As experiments results, we have data of 13 games, 13 sets of start-to-end situations $\left\{\left(s_{1}(1), s_{2}(1), s_{3}(1)\right), \ldots,\left(s_{1}\left(t_{g}^{e}\right), s_{2}\left(t_{g}^{e}\right), s_{3}\left(t_{g}^{e}\right)\right)\right\}$, where $t_{g}^{e}$ is the end step of game $g \in\{1, \ldots, 13\}$. Of course, in addition to situations, we also have derived data: given resources, transfers, and profits in accordance with the $\mathrm{YH}$ mechanism.

\section{The Main Approach: Nash Bargaining Solution}

We can treat the game process as a negotiation process among players: they bargain their profits. If they have reached a satisfactory result, they do not have a desire to change anything and therefore, will not change bids, and the game will stop by the "stop rule".

\subsection{The Zeuthen-Hicks Bargaining Model}

Initial Zeuthen-Hicks bargaining model considers the interaction between two parties - seller and buyer (Harsanyi, 1956; Vetschera, 2018).

The model considers negotiations between two parties 1 and 2. Denote an arbitrary party as $i \in N=\{1,2\}$, and $i$ 's opponent by $(-i)$. The current offer of party $i$ is $x_{i}$.

The utility function of party $i$ is $u_{i}(x)$. At each step, party $i$ has to decide whether to accept the offer $x_{-i}$ of the opponent or insist on its own offer $x_{i}$, which can be accepted or rejected by the opponent. Rejection by the opponent leads to termination of the negotiation. In that case, each party receives a disagreement utility of $d_{i}$.

The probability that opponent will reject offer $x_{i}$ is denoted by $p_{-i}$. Party $i$ will accept the opponent's offer if

$$
u_{i}\left(x_{-i}\right)>p_{-i} u_{i}\left(d_{i}\right)+\left(1-p_{-i}\right) u_{i}\left(x_{i}\right) .
$$

It's supposed that hold

$$
u_{i}\left(d_{i}\right)<u_{i}\left(x_{-i}\right) \leq u_{i}\left(x_{i}\right) .
$$


From (7), one can determine a critical probability $p_{-i}^{*}$ at which party $i$ is indifferent between accepting and making a counteroffer:

$$
p_{-i}^{*}=\frac{u_{i}\left(x_{i}\right)-u_{i}\left(x_{-i}\right)}{u_{i}\left(x_{i}\right)-u_{i}\left(d_{i}\right)} .
$$

If $p_{-i}>p_{-i}^{*}$, it is better for $i$ to accept the opponent's offer rather than insist on $x_{i}$. Therefore, $p_{-i}^{*}$ can be considered as a measure of the strength of party $i$ in the current state $\left(x_{i}, x_{-i}\right)$ of the negotiation. The weaker party will then make a concession. Thus party $i$ makes a concession if $p_{-i}^{*}<p_{i}^{*}$ which after substitution (9) is equivalent to

$$
\left(u_{i}\left(x_{i}\right)-u_{i}\left(d_{i}\right)\right)\left(u_{-i}\left(x_{i}\right)-u_{-i}\left(d_{-i}\right)\right)<\left(u_{i}\left(x_{-i}\right)-u_{i}\left(d_{i}\right)\right)\left(u_{-i}\left(x_{-i}\right)-u_{-i}\left(d_{-i}\right)\right) .
$$

Therefore, the currently weaker party will make a concession to revert the inequality sign in (10) and thus has an incentive to increase the value of the Nash bargaining objective function

$$
U(x)=\left(u_{i}(x)-u_{i}\left(d_{i}\right)\right) \cdot\left(u_{-i}(x)-u_{-i}\left(d_{-i}\right)\right)
$$

the maximum of which is the Nash bargaining solution (Nash, 1950).

\subsection{The Nash Bargaining Solution (NBS)}

In case of three players it is not possible to treat negotiation as Zeuthen-Hicks Bargaining, but we can look at the Nash Bargaining objective function:

$$
U^{N a s h}(s, d)=\prod_{i=1}^{3}\left(\phi_{i}(s)-u_{i}\left(d_{i}\right)\right) \underset{x \in A}{\longrightarrow} \max ,
$$

where $d=(0,0,0)$ with $u(d)=(1,3,5)$, i.e. disagreement utility is base utility without transfers $p_{i}=0$ and resources $x_{i}=0$.

Due to YH mechanism, we use a profit $\phi$ of players instead of their utilities. Value of $U^{\text {Nash }}(s, d)$ in a concrete situation we will call "NBS value" for simplicity.

Another way to check significance of NBS to players is "local NBS" version:

$$
U_{l o c}^{N a s h}(s, t)=\prod_{i=1}^{3}\left(\phi_{i}(s)-\phi_{i}(s(t-1)) \underset{x \in A}{\longrightarrow} \max .\right.
$$

In our concrete case, $U^{\text {Nash }}(s, d)$ and moreover $U_{l o c}^{\text {Nash }}(s, t)$ can be positive if two of three multipliers in (12) and (13) are negative. Therefore we use "shifted" objective functions:

$$
\begin{aligned}
U(s, d) & =\min _{i} \operatorname{sign}\left(\phi_{i}(s)-u_{i}\left(d_{i}\right)\right) \cdot\left|U^{N a s h}(s, d)\right| \\
U_{l o c}(s, t) & =\min _{i} \operatorname{sign}\left(\phi_{i}(s)-\phi_{i}(s(t-1))\right) \cdot\left|U_{l o c}^{N a s h}(s, t)\right| .
\end{aligned}
$$

The (14) and (15) functions are positive only if all multipliers (12) and (13) are positive. Additionally, for local NBS it means that player's profits have increased at step $t$.

Now let us proceed to the description of our approach to behavior analysis. 


\section{Behavior Models}

We estimate the shares of decisions made by players that correspond to some behavior models and the shares of situations when some or all players made decisions that correspond to some behavior models.

Let's denote $c$ - the count of all individual bids in our data, $C$ - the count of all situations. For our 3-player games considered $c=3 C$. Then the share of some behavior ' $B$ ' is $\#\left\{s_{i}(t) \mid s_{i}(t) \in B\right\} / c$ and the share of some set of situations ' $P$ ' is $\#\{s(t) \mid s(t) \in P\} / C$.

Now let us proceed to the description of the behavior models under consideration.

\section{Nash bargaining behavior}

- Firstly we consider decisions that go into direction of NBS increase, independently of whether the move is sufficiently large. We treat $s_{i}(t)$ to be Nashincreasing (NI) decision if:

$$
U\left(s^{i}(t), d\right)>U(s(t-1), d), i \in N
$$

where $s^{i}(t)=\left(s_{i}(t), s_{-i}(t-1)\right)$ and $d=(0,0,0)$ :

- Further we can define Real Nash increasing (Real NI) situations. We treat $s(t)$ to be Real Nash-increasing situation if it did increase the NBS value at step $t$ :

$$
U(s(t), d)>U(s(t-1), d) .
$$

- Similarly instead of NBS we can use local NBS. We treat $s_{i}(t)$ to be local Nashincreasing (LNI) decision if:

$$
U_{l o c}\left(s^{i}(t), t\right)>0
$$

- and we treat $s(t)$ to be Real local Nash-increasing (Real LNI) situation if:

$$
U_{l o c}(s(t), t)>0
$$

Rational behavior All rational behavior models are based on the best response (BR) of a player to a situation on the previous step:

$$
b r_{i}(s(t-1))=\underset{y \in \mathbb{R}^{+}}{\arg \max } \phi_{i}\left(y_{i}, s_{-i}(t-1)\right) .
$$

Let's consider two rational behavior models.

- We treat $s_{i}(t)$ to be near best response with accuracy $\varepsilon(\operatorname{BR}(\varepsilon))$ if:

$$
\left|s_{i}(t)-b r_{i}(s(t-1))\right|<\varepsilon
$$

- We treat $s_{i}(t)$ to be Toward $B R(\mathrm{TBR})$ if:

$$
\left\{\begin{array}{l}
s_{i}(t)=s_{i}(t-1), \text { if } b r_{i}(s(t-1))=s_{i}(t-1) \\
\left(s_{i}(t)-s_{i}(t-1)\right) /\left(b r_{i}(s(t-1))-s_{i}(t-1)\right)>0, \text { otherwise. }
\end{array}\right.
$$


Constant behavior We treat some sequence of bids of one player $i$ from step $t_{s}$ to step $t_{e}>t_{s}$ with accuracy $\varepsilon$ to be constant behavior $C B\left(i, t_{s}, t_{e}, \varepsilon\right)$ if:

1. $\left|s_{i}\left(t_{s}, t_{e}\right)\right| \leq \varepsilon$

2. $\forall a<t_{s}, b>t_{e}:\left|s_{i}\left(a, t_{e}\right)\right|>\varepsilon \wedge\left|s_{i}\left(t_{s}, b\right)\right|>\varepsilon$

3. Not exists another $C B\left(i, t_{s}^{\prime}, t_{e}^{\prime}, \varepsilon\right)$ such that $t_{s}^{\prime}<t_{s}$ and $t_{s}<t_{e}^{\prime}<t_{e}$.

The above items describe mathematically results of the "algorithm" of the sequential search for unchanged bids (with some accuracy $\varepsilon$ ): starting from the first step, we are looking for the bids sequence of players whose maximum and minimum differ by no more than $\varepsilon$.

The set of all CB with accuracy $\varepsilon$ is denoted as $C B(\varepsilon)$.

- We treat $s_{i}(t)$ to be Agree $C B(\mathrm{ACB})$ if a player doesn't change his decision at all - signal that he is agree with allocation:

$$
s_{i}(t)=s_{i}(t-1)
$$

- We treat $s_{i}(t)$ to be Waiting $C B$ (WCB) if a player slightly changes his decision in order not to stop negotiation process:

$$
W C B(\varepsilon)=C B(\varepsilon) \backslash A C B
$$

- We treat $s_{i}(t)$ to be Rational $W C B($ RWCB) if a player perform WCB toward his $\mathrm{BR}$ :

$$
R W C B(\varepsilon)=W C B(\varepsilon) \cap T B R
$$

\section{Results}

\subsection{Individual Decisions in YH Games}

In the table below (see figure 1), the numbers and shares of individual decisions that correspond to behavior models described above are presented. In the left and right parts of the table are depicted results about all games, and games ended with consensus. We have only three consensus-ended games, but they contain 56 situations $(31,19$, and 6$)$ with 168 individual decisions. Rows with couple behavior models, separated by $\backslash$ (for example - LNI $\backslash \mathrm{NI}$ ), correspond to decisions that suit a first behavior model but not to a second one.

The most observed models in all games are TBR, Agree CB, and Nash increasing. The data of games with consensus is similar to all games, but there is an exceptions:

1. Local Nash increasing models are found twice more often, and all such decisions are rational,

2. Nash increasing models without CB have grown,

3. less Waiting and Rational Waiting CB decisions,

4. $\mathrm{BR}(1)$ is doubled from $5 \%$ to $10 \%$, but TBR does not change.

So most observed models in all games and consensus-ended games are almost equivalent, but other models are changed. In consensus-ended games, players exhibit more intention to act accordingly to local NBS, i.e., to increase utility of all players. It seems that non-Agree $\mathrm{CBs}$ occur significantly rare. Item 4 maybe shows that TBR decisions in consensus games are more concentrated near BR with accuracy 1 than in all games. 


\begin{tabular}{|c|c|c|c|c|}
\hline & \multicolumn{2}{|r|}{ All games } & \multicolumn{2}{|c|}{ Games with consensus } \\
\hline & num & $\%$ & num & $\%$ \\
\hline games & 13 & & 3 & \\
\hline total iterations & 326 & & 56 & \\
\hline \multirow[t]{2}{*}{ total decisions } & 978 & $100 \%$ & 168 & $100 \%$ \\
\hline & \multicolumn{4}{|c|}{ Rational behavior } \\
\hline $\mathrm{BR}(1)$ & 47 & $5 \%$ & 16 & $10 \%$ \\
\hline \multirow[t]{2}{*}{ Toward BR } & 435 & $44 \%$ & 74 & $44 \%$ \\
\hline & \multicolumn{4}{|c|}{ Constant Behavior } \\
\hline Agree CB & 295 & $30 \%$ & 55 & $33 \%$ \\
\hline Waiting $C B(1) \backslash A C B$ & 120 & $12 \%$ & 5 & $3 \%$ \\
\hline \multirow[t]{2}{*}{ Rational WCB(1) } & 74 & $8 \%$ & 4 & $2 \%$ \\
\hline & \multicolumn{4}{|c|}{ Nash Bargaining Solution Behavior } \\
\hline $\mathrm{NI}$ & 311 & $32 \%$ & 53 & $32 \%$ \\
\hline $\mathrm{NI} \backslash W C B(1)$ & 253 & $26 \%$ & 52 & $31 \%$ \\
\hline Rational $\mathrm{NI} \backslash W C B(1)$ & 161 & $16 \%$ & 40 & $24 \%$ \\
\hline Local NI & 110 & $11 \%$ & 37 & $22 \%$ \\
\hline LNI \WCB(1) & 109 & $11 \%$ & 37 & $22 \%$ \\
\hline Rational LNI\WCB(1) & 109 & $11 \%$ & 37 & $22 \%$ \\
\hline LNI\NI & 16 & $2 \%$ & 3 & $2 \%$ \\
\hline
\end{tabular}

Fig. 1. Individual decisions in all $\mathrm{YH}$ games and in consensus-ended YH games.

\subsection{Decision Situations}

In the previous section, we see at bids of players and their intention toward BR and NI, but to see at dynamic of negotiation process we can see at situations - behavior of player's group altogether $s=\left(s_{1}, s_{2}, s_{3}\right)$. We consider two types of situations: when all players act according to the same behavior model and when at least one player acts according to it.

In figure 2 Rational and Constant behavior models are presented. Games with consensus have most differences again with less Waiting CB behaviors. Most observed "at least one TBR" and "at least one Agree CB" again do not have significant changes. "At least One BR(1)" again has more percent in consensus games (almost three times), but TBR situations do not change.

In figure 3 NBS models in situations are considered. Real increase of Nash function relates to 'All ...' models and has the most observable cases in the class. It turns out that about half of situations did increase Nash value! Count of real Nash increasing situations with at least one player toward Nash increasing equal to 155, with at least two players equal to 67 .

So real Nash increasing also occur in 88 (155-67) situations when only one of players makes decision toward Nash increasing. Maybe other players do not change their bids (remember Agree CB is $30 \%$ of individual decisions), but it is an interesting question about what other situations can real increase Nash function.

Concerning differences with consensus games, again (as with individual decisions), we see doubled count of situations with observed Local NI models. So when considering situations, we see that players who demonstrate wish to increase local 


\begin{tabular}{|c|c|c|c|c|}
\hline & \multicolumn{2}{|c|}{ All games } & \multicolumn{2}{|c|}{ Games with consensus } \\
\hline & num & $\%$ & num & $\%$ \\
\hline games & 13 & & 3 & \\
\hline \multirow[t]{2}{*}{ total iterations } & 326 & $100 \%$ & 56 & $100 \%$ \\
\hline & \multicolumn{4}{|c|}{ Rational behavior } \\
\hline All BR(1) & 0 & $0 \%$ & 0 & $0 \%$ \\
\hline at least One BR(1) & 44 & $13 \%$ & 17 & $30 \%$ \\
\hline All TBR & 29 & $9 \%$ & 6 & $11 \%$ \\
\hline \multirow[t]{2}{*}{ at least One TBR } & 273 & $84 \%$ & 46 & $82 \%$ \\
\hline & \multicolumn{4}{|c|}{ Constant Behavior } \\
\hline All Agree CB & 3 & $1 \%$ & 3 & $5 \%$ \\
\hline at least One ACB & 215 & $66 \%$ & 40 & $71 \%$ \\
\hline All Waiting $\mathrm{CB}(1)$ & 1 & $0 \%$ & 0 & $0 \%$ \\
\hline at least One WCB(1) & 111 & $34 \%$ & 5 & $9 \%$ \\
\hline All Rational WCB & 0 & $0 \%$ & 0 & $0 \%$ \\
\hline at least One RWCB & 70 & $21 \%$ & 4 & $7 \%$ \\
\hline
\end{tabular}

Fig. 2. Decision situations in all YH games and in consensus-ended YH games.

Nash function (i.e., utilities) came to an agreement. Alternatively, consensus-ended games have more players who demonstrate wish to increase utilities.

In figure 4 count of steps until the end of games from the last observed model are presented.

In games with consensus, we see fewer values on average compared to timeoutended games. 'All ...' decisions rare belongs to the second half of game length, but 'One ...', Real NI, and Real LNI situations are near the end of game, especially to consensus games. This data tells us that in consensus games last of NBS behavior observed close to end. It can be treated as NBS triggers the end of negotiation process. Interestingly, some short games with $T \leq 20$ do not have situations 'All NI' and 'All LNI', unlike games with $T=60$ or consensus.

An example of negotiation process for one game ended with consensus is presented in figure 5. At steps 2 and 5 individual decisions and situations are NBS

\begin{tabular}{|c|c|c|c|c|}
\hline & \multicolumn{2}{|c|}{ All games } & \multicolumn{2}{|c|}{ Games with consensus } \\
\hline & num & $\%$ & num & $\%$ \\
\hline games & 13 & & 3 & \\
\hline \multirow[t]{2}{*}{ total iterations } & 326 & $100 \%$ & 56 & $100 \%$ \\
\hline & \multicolumn{2}{|c|}{ Nash Bargaining Solution Behavior } & & \\
\hline All NI & 11 & $3 \%$ & 4 & $7 \%$ \\
\hline at least One NI & 224 & $69 \%$ & 35 & $63 \%$ \\
\hline Real NI & 156 & $48 \%$ & 25 & $45 \%$ \\
\hline Real NI \& One NI & 155 & $48 \%$ & 25 & $45 \%$ \\
\hline Real NI \& Two NI & 67 & $21 \%$ & 13 & $23 \%$ \\
\hline All Local NI & 7 & $2 \%$ & 3 & $5 \%$ \\
\hline at least One LNI & 75 & $23 \%$ & 24 & $43 \%$ \\
\hline Real LNI & 49 & $15 \%$ & 17 & $30 \%$ \\
\hline
\end{tabular}

Fig. 3. Decision situations in YH games, NBS models. 


\begin{tabular}{|c|c|c|c|c|c|c|c|c|}
\hline \multirow[b]{3}{*}{ Game } & \multirow[b]{3}{*}{ length } & \multirow[b]{3}{*}{$\begin{array}{l}\text { end of } \\
\text { game }\end{array}$} & \multicolumn{6}{|c|}{ Type of event } \\
\hline & & & \multicolumn{4}{|c|}{ Descisions } & \multicolumn{2}{|c|}{ Situation } \\
\hline & & & All NI & One NI & All LNI & One LNI & $\begin{array}{c}\text { Real } \\
\mathrm{NI}\end{array}$ & $\begin{array}{c}\text { Real } \\
\text { LNI }\end{array}$ \\
\hline 40 & 60 & timeout & 11 & 2 & 45 & 6 & 2 & 2 \\
\hline 41 & 19 & consensus & 8 & 1 & 17 & 4 & 1 & 4 \\
\hline 42 & 6 & consensus & 4 & 1 & 4 & 1 & 1 & 1 \\
\hline 43 & 60 & timeout & 36 & 0 & 18 & 36 & 1 & 40 \\
\hline 56 & 31 & consensus & 16 & 2 & 15 & 2 & 2 & 2 \\
\hline 57 & 60 & timeout & 19 & 1 & 57 & 4 & 3 & 4 \\
\hline 60 & 15 & timeout & none & 0 & none & 1 & 1 & none \\
\hline 61 & 15 & timeout & none & 1 & none & 4 & 2 & 4 \\
\hline 92 & 20 & timeout & 6 & 1 & none & 15 & 1 & 6 \\
\hline 93 & 20 & timeout & 14 & 0 & 14 & 9 & 2 & 14 \\
\hline 94 & 20 & timeout & none & 0 & none & 5 & 1 & 4 \\
\hline
\end{tabular}

Fig. 4. NBS and end of games.

agree, or as at step 5, two players make LNI bids, and one does not change bid at this step and further, signaling about satisfaction. May be players act cooperatively, and these characteristics of situations at steps 2 and 5 may be indicators of cooperative behavior.

\section{Conclusion}

The research conducted allows us to conclude that indicators based on Nash bargaining value do allow us to predict possibilities for negotiation parties to reach a consensus.

The initial design of experiments under consideration was not intended to identify if some participants behave toward Nash Bargaining Solution. Analysis of data shows us some evidence that a significant part of decisions and situations suit behavior that leads to an increase of NBS value as opposed to best response behavior. Nevertheless, there was no evidence found that increase of some "global" NBS value may be somehow connected with the possibility to reach the consensus. However, switching attention to a local increase of NBS in the style of (Vetschera, 2018) turns out to be fruitful.

In cases when parties reached an agreement, all participants of such games took more decisions to increase local Nash Bargaining value. Furthermore, the last situations of real Nash function increasing were encountered almost at the end of games.

\begin{tabular}{|r|l|l|l|r|r|l|}
\hline Iteration & Player 1 & Player 2 & Player 3 & NI & LNI & \\
\hline 6 & ACB & ACB & ACB & 0 & 0 & game ended \\
\hline & LNI\& & & LNI\& & & & \\
& NI\&TBR & ACB & NI\&TBR & 1 & 1 & cooperation? \\
\hline 4 & $?$ & RWCB & TBR & 0 & 0 & \\
\hline 3 & TBR & NI\&TBR & ACB & 1 & 0 & \\
\hline & LNI\& & LNI\& & LNI\& & & & \\
2 & NI\&TBR & NI\&TBR & NI\&TBR & 1 & 1 & cooperation? \\
\hline
\end{tabular}

Fig. 5. Negotiation example. 
At the same time, it should be pointed out that such local increment of Nash Bargaining function while achieving some consensus may be quite far from Nash Bargaining Solution. However, taking in to account the fact that particular NBS depends on selection of disagreement solution, while local increase of Nash Bargaining function as it is defined here and in (Vetschera, 2018) depends only on situation at previous iteration of bargaining process.

The perspectives of the further directions of research following the approach described in this paper may be suggested. The first obvious direction is to extend the analysis conducted on data from experimental games with other mechanisms described in (Korgin and Korepanov, 2017). The main difficulty is to define NBS behavior with multidimensional signals, like in GL mechanism. The next direction is connected with the concept of reflexion or strategic thinking (Chkartishvili and Korepanov, 2016) - if the knowledge about Nash function and NBS will affect the decision-making process of parties during negotiation or not. Finally, redesign of experiment to identify if participants think about something in common with Nash Bargaining value should be conducted to verify all the hypotheses developed through previous stages.

\section{Appendix}

\section{Yang-Hajek's proportional mechanism}

The Yang-Hajek's class of mechanisms (Yang and Hajek, 2005) is one of the classes of resource allocation mechanisms developed to deal with the incentive incompatibility. In our research we used a mechanism from this class with the following parameters.

Action of each player - amount of resource she would like to receive - bid $s_{i} \in \mathbb{R}_{+}$. All players gives their bids $s=\left(s_{1}, s_{2}, s_{3}\right)$. Denote $S=\sum_{j} s_{j}$.

Resource that will be given to player $i$ is equal to $x_{i}=R * s_{i} / S$. Transfer of player $\tau_{i}=\beta s_{i}\left(S-s_{i}\right)$, where $\beta=0.0005$ - penalty strictness.

And player's profit is $\phi_{i}=u_{i}\left(x_{i}\right)-\tau_{i}$.

\section{References}

Başar, T. and Maheswaran, R. T. (2003). Nash equilibrium and decentralized negotiation in auctioning divisible resources. Group Decision and Negotiation, 12(5), 361-395.

Boyd, S., Parikh, N., Chu, E. (2011). Distributed Optimization and Statistical Learning via the Alternating Direction Method of Multipliers. Foundations and Trends in Machine Learning, 3(1), 1-122.

Chkhartishvili, A. G., Korepanov, V.O. (2016). Adding informational beliefs to the players strategic thinking model. IFAC-PapersOnLine, 49(32), 19-23.

Groves, T., Ledyard, J.O. (1977). Optimal allocation of public goods: A solution to the 'freerider' problem. Econometrica, 45, 783-809.

Groves, T., Ledyard, J. O. (1985). Incentive compatibility ten years later. Discussion Papers, 648.

Harsanyi, J. C. (1956). Approaches to the bargaining problem before and after the theory of games: A critical discussion of Zeuthen's, Hicks', and Nash's theories. Econometrica, 24(2), 144-157.

Korgin, N., Korepanov, V. (2016) An Efficient Solution of the Resource Allotment Problem with the Groves-Ledyard Mechanism under Transferable Utility. Automation and Remote Control, 77(5), 914-942. 
Korgin, N., Korepanov, V. (2017) Experimental gaming comparison of resource allocation rules in case of transferable utilities. International Game Theory Review, 19(2), 1750006.

Nash, J. F. (1950). The bargaining problem. Econometrica, 18(2), 155-162.

Yang, S., Hajek, B., (2005). Revenue and stability of a mechanism for efficient allocation of a divisible good, mimeo. Urbana Champaign: University of Illinois, $35 \mathrm{p}$.

Vetschera, R. (2018). Zeuthen-Hicks Bargaining in Electronic Negotiations. Group Decision and Negotiation, 28, 255-274. 